are associated with reduced therapeutic efficacy as compared to RA patients with normal body composition. The unfavourable effect of body weight on disease activity was paralleled by a favourable, protective effect on joint destruction. This uncoupling is not fully elucidated yet, and should be further investigated.

Disclosure of Interest: None declared

DOI: 10.1136/annrheumdis-2017-eular.4694

\section{OP0151 DOCOSAHEXAENOIC ACID TREATMENT OF RHEUMATOID ARTHRITIS: A RANDOMIZED, DOUBLE-BLIND, PLACEBO-CONTROLLED, CROSS-OVER STUDY}

T. Neumann ${ }^{1,2}$, C. Dawczynski ${ }^{3,4}$, M. Dittrich ${ }^{5}$, K. Goetze ${ }^{6}$, A. Welzel ${ }^{1}$, P. Oelzner ${ }^{1}$, S. Voelker ${ }^{7}$, A.M. Schaible ${ }^{7}$, F. Troisi ${ }^{7}$, L. Thomas ${ }^{7}$, S. Pace ${ }^{7}$ A. Koeberle ${ }^{7}$, O. Werz ${ }^{7}$, P. Schlattmann ${ }^{8}$, S. Lorkowski ${ }^{3,4}$, G. Jahreis ${ }^{3,4}$.

${ }^{1}$ Department of Internal Medicine III, Jena University Hospital, Jena, Germany;

${ }^{2}$ Department of Rheumatology, Kantonsspital St. Gallen, St. Gallen, Switzerland;

${ }^{3}$ Competence Cluster for Nutrition and Cardiovascular Health (nutriCARD);

${ }^{4}$ Department of Nutritional Biochemistry and Physiology, Institute of Nutrition;

${ }^{5}$ Department of Nutritional Physiology, Institute of Nutrition, Friedrich Schiller

University of Jena; ${ }^{6}$ Department of Rheumatology, Jena University Hospital;

${ }^{7}$ Department of Pharmaceutical/Medicinal Chemistry; ${ }^{8}$ Department of Medical

Statistics, Informatics and Documentation, Friedrich Schiller University of Jena,

Jena, Germany

Background: Epidemiological studies and clinical investigations indicate a beneficial impact of long-chain n-3 polyunsaturated fatty acids ( $n-3$ PUFA) on the inflammatory activity of rheumatoid arthritis (RA). However, the knowledge about the physiological effects of the individual compounds eicosapentaenoic acid (EPA) and docosahexaenoic acid (DHA) is limited.

Objectives: In our pilot study presented here, we investigated the clinical benefit of daily intake of foods enriched with microalgae oil as source of DHA in RA patients. In particular, the influence on disease activity and changes in the profile of pro-inflammatory/non-resolving and anti-inflammatory/pro-resolving lipid mediators was examined.

Methods: This is a randomized, double-blind, cross-over study on 38 patients (32 females/ 6 males) with active RA (DAS28 $\geq 2.4$ ). They were allocated to consume foods enriched with microalgae oil from Schizochytrium $s p$. (2.1 g DHA/day) or sunflower oil (placebo) for 10 weeks while maintaining stable immunosuppressive treatment.

Results: Thirty-two patients finished the study but seven patients were excluded from analysis because their DHA increase in erythrocyte lipids (EL) was less than $25 \%$ after 10 weeks of intervention indicating insufficient adherence to the intervention. Supplementation of DHA led to a decline in tender joints (TJ68) $8.4+5.6$ to $6.0+5.0(\mathrm{p}=0.03)$, swollen joints (SJ66) $5.6 \pm 3.5$ to $3.9 \pm 3.5(\mathrm{p}=0.07)$ and DAS28 $4.3 \pm 1.0$ to $3.9 \pm 1.2(p=0.07)$. Joint counts in the placebo arm remained stable (TJ68) $6.7 \pm 4.9$ to $8.8 \pm 8.0 \quad(p=0.12)$, swollen joints (SJ66) $3.5 \pm 3.0$ to 4.2 $\pm 3.7(\mathrm{p}=0.40)$ and DAS28 $4.0 \pm 0.9$ to $4.1 \pm 1.2(\mathrm{p}=0.45)$. Ultrasound score (US-7) remained stable ( $15.1 \pm 9.5$ to $12.4 \pm 7.0 ; p=0.160)$ while it increased in the placebo arm $(11.4 \pm 7.0$ to $14.0 \pm 8.8 ; p=0.03)$.

The amount of $\mathrm{n}-3$ PUFA in erythrocyte increased in supplemented patients ( $7.0 \pm 1.2 \%$ to $10.6 \pm 1.4 \% ; p<0.001)$ and the ratios of aracidonic acid (AA) /EPA (18.5 \pm 6.3 to $12.6 \pm 5.5 ; p<0.001)$ and $A A / D H A(3.8 \pm 1.0$ to $1.8 \pm 0.6 ; p<0.001)$ dropped significantly. Fatty acid distribution in erythrocyte lipids (n-3 PUFA, $A A / E P A$ and $A A / D H A)$ remained stable in the control arm.

Conclusions: The study shows that microalgae DHA supplements can ameliorate disease activity in patients with active RA along with a shift in the balance of AA- and DHA-derived lipid mediators towards an anti-inflammatory/pro-resolving state.

Disclosure of Interest: None declared

DOI: 10.1136/annrheumdis-2017-eular.6759

\section{OP0152 A LONGITUDINAL STUDY OF THE EFFECTS OF DISEASE ACTIVITY ON RENAL FUNCTION IN PATIENTS WITH RHEUMATOID ARTHRITIS UTILIZING LINEAR MIXED EFFECT MODELS - ANSWER COHORT STUDY -}

A. Onishi ${ }^{1}$, K. Akashi ${ }^{1}$, M. Hashimoto ${ }^{2}$, M. Furu ${ }^{2}$, W. Yamamoto ${ }^{2}$, M. Katayama ${ }^{3}$, R. Hara ${ }^{4}$, T. Fujimura ${ }^{4}$, S. Yoshida ${ }^{5}$, K. Nagai ${ }^{5}$, T. Hirano ${ }^{6}$, K. Ebina ${ }^{7}$, Y. Son ${ }^{8}$, H. Amuro ${ }^{8}$, H. Yamada ${ }^{1}$, Y. Ichise ${ }^{1}$, D. Waki ${ }^{1}$, I. Naka ${ }^{1}$, K. Tsuda ${ }^{1}$, T. Okano ${ }^{1}$, S. Takahashi ${ }^{1}$, S. Sendo ${ }^{1}$, Y. Ueda ${ }^{1}$, Y. Kogata ${ }^{1}$, J. Saegusa ${ }^{1}$, A. Morinobu ${ }^{1} .{ }^{1}$ Department of Rheumatology, Kobe University Hospital, Kobe; ${ }^{2}$ Department of the Control for Rheumatic diseases, Graduate School of Medicine, Kyoto University, Kyoto; ${ }^{3}$ Department of Rheumatology and Clinical Immunology, Osaka Red Cross Hospital, Osaka; ${ }^{4}$ The Center for Rheumatic Diseases, Nara Medical University, Nara; ${ }^{5}$ Department of Internal Medicine (I), Osaka Medical College; ${ }^{6}$ Department of Respiratory Medicine, Allergy and Rheumatic Disease, Graduate School of Medicine, Osaka University; ${ }^{7}$ Department of Orthopaedic Surgery, Osaka University, Graduate School of Medicine: ${ }^{8}$ First Department of Internal Medicine, Kansai Medical University, Osaka, Japan

Background: Patients with RA have higher risk of developing chronic kidney disease compared to the general population. Although some causes of renal impairment in RA include nephrotoxic medications, amyloidosis, and glomerulonephritis, specific causes are not determined among most patients with RA. While experimental researches showed inflammatory process per se might also contribute to renal dysfunction, the results of previous clinical studies that assessed the effects of disease activity or C-reactive protein on renal function were inconsistent. This inconsistency might be attributable to their cross-sectional design, small sample size, and assessment of association between only baseline characteristics and renal outcomes without consideration for the fact that disease activity and medications had changed over time.

Objectives: To identify the effects of disease activity on renal function in RA in a multi-center cohort study.

Methods: RA patients with a sampling interval of less than 150 days were enrolled because wide sampling intervals could not take into consideration changes in disease activity and medications during their follow-up. An estimated glomerular filtration rate (eGFR) was calculated using an equation approved by the Japanese Society of Nephrology and used as an outcome variable. Linear mixed effect models were used to evaluate the renal trajectories of patients. Time from baseline (months), disease activity, and their interaction were included as fixed effects while participant identification number and time from baseline were included as random factors. Age, sex, disease duration, RF, ACPA, NSAIDs, and DMARDs that were known as a cause of renal impairment, such as tacrolimus, iguratimod, and tofacitinib, were included as covariates.

Results: A total of 25661 samples (mean sampling interval: 2.0 months) from 2104 patients was included. Patients with lower DAS28-CRP had worse renal function at inclusion, but a significantly better longitudinal trajectory on eGFR $\left(0.0079 \mathrm{ml} / \mathrm{min} / 1.73 \mathrm{~m}^{2}\right.$ per month, $\left.\mathrm{P}=0.025\right)$. Although all $\mathrm{RA}$ patients had naturally progressive renal impairment as they got older, patients who achieved remission or low disease activity had slower renal impairment rate of -0.068 $\mathrm{ml} / \mathrm{min} / 1.73 \mathrm{~m}^{2}$ per month compared to patients with moderate or high disease activity $\left(-0.084 \mathrm{ml} / \mathrm{min} / 1.73 \mathrm{~m}^{2}\right.$ per month; $\left.\mathrm{P}=0.037\right)$. These results were also similar using SDAI or CDAI.

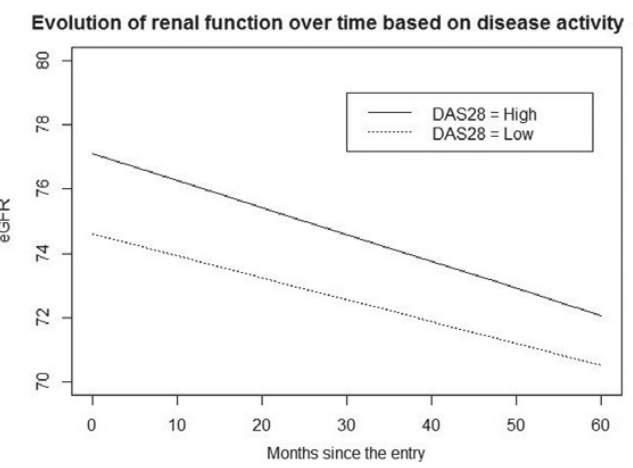

Conclusions: Lower disease activity results in slower renal impairment. Because the effects of disease activity on renal function are mild, additional measures to protect renal function, such as avoiding nephrotoxic medications and treating cardiovascular risk factors are important.

Disclosure of Interest: None declared DOI: 10.1136/annrheumdis-2017-eular.4544

\section{OP0153 RECURRENT INFECTIONS IN RHEUMATOID ARTHRITIS PATIENTS, RESULTS FROM THE BSRBR}

S. Subesinghe ${ }^{1}$, A.I. Rutherford ${ }^{1}$, R. Byng-Maddick ${ }^{2}$, J.B. Galloway ${ }^{1}$. ${ }^{1}$ Rheumatology, King's College London; ${ }^{2}$ Homerton Hospital, London, United Kingdom

Background: Rheumatoid arthritis (RA) patients have an increased susceptibility to infection.

Objectives: 1.To establish the rate of recurrent infection in RA patients recruited to the British Society of Rheumatology Biologics Registry Rheumatoid Arthritis (BSRBR-RA).2.To establish whether the organ class of index infection predicted future serious infection.

Methods: The BSRBR-RA is a prospective observational cohort, previously described. Patients with at least one episode of serious infection requiring hospitalisation were included if they occurred whilst on anti-rheumatic drug therapy or within 5 drug half-lives of stopping. Infections were coded by MedDRA classification in to 7 categories. Infections occurring over 14 days after the first index infection were considered as new events. Event rates were calculated and compared using a Cox proportional hazards model with adjustments made for age, gender, disease duration, baseline DAS28 score, smoking status and seropositivity.

Results: See Table 1.

In total, 21,943 subjects with 115,423 patient-years follow up were studied, 5365 subjects reported at least one serious infection. Comparing organ classes of prior infection at baseline, each group had comparable age, disease duration, baseline DAS28 and HAQ scores. The cohort characteristics are tabulated. The baseline annual rate of first serious infection was $4.6 \%(95 \% \mathrm{Cl} 4.5-4.7)$. Following an 